\title{
Lack of Increased Risk of Lymphoma with Thiopurine Therapy Regardless of Dose and Duration of Treatment in Japanese Patients with Inflammatory Bowel Diseases
}

\author{
Taku Kobayashi $^{\mathrm{a}}$ Eri Udagawa ${ }^{\mathrm{b}}$ Toshifumi Hibi ${ }^{\mathrm{a}}$ \\ ${ }^{a}$ Center for Advanced IBD Research and Treatment, Kitasato University Kitasato Institute Hospital, Minato-ku, Japan; \\ bJapan Medical Office, Takeda Pharmaceutical Company Limited, Chuo-ku, Japan
}

\section{Keywords}

Thiopurine $\cdot$ Inflammatory bowel disease $\cdot$ Lymphoma risk

\begin{abstract}
Background: Immunomodulator therapy (e.g., thiopurines) has been linked to an increased malignancy risk, in patients with inflammatory bowel diseases (IBDs), which increases with treatment duration, based on studies mainly in Caucasian patients. However, our previous real-world study, of Japanese patients with IBDs, indicated no overall increased risk of non-Hodgkin lymphoma (NHL) with thiopurine treatment. Objectives: This subanalysis investigated the influence of thiopurine IBD treatment dose and duration, on incidence of NHL in Japanese patients. Method: The Medical Data Vision (MDV) claims database (17.8 million patients; April 2008-January 2018) was used to analyze incidence rate ratios (IRRs) of $\mathrm{NHL}$, in eligible patients ( $\geq 1$ diagnosis of $\mathrm{ul}$ cerative colitis or Crohn's disease) and no malignancy at diagnosis or first prescription of a thiopurine. Age- and sexadjusted IRRs and 95\% confidence interval for NHL were calculated as the incident cases compared in the subgroups versus the overall IBD population. Results: Among 75,673 patients with IBDs, 103 cases of NHL were recorded. There was no overall increase in the risk of developing NHL among Japanese patients treated with thiopurines. The IRRs relative
\end{abstract}

karger@karger.com www.karger.com/dig

Karger $\stackrel{\text { ' }}{5}$

GOPEN ACCESS
(C) 2022 The Author(s)

Published by S. Karger AG, Basel

This is an Open Access article licensed under the Creative Commons Attribution-NonCommercial-4.0 International License (CC BY-NC) (http://www.karger.com/Services/OpenAccessLicense), applicable to the online version of the article only. Usage and distribution for commercial purposes requires written permission. to the overall IBD population were $1.88,1.42$, and 0.38 for $<1$ year, $1-3$ years, and $\geq 3$ years of thiopurine treatment. There were no differences in NHL incidence when grouping patients by mean daily thiopurine dose prescribed, age, or disease subgroups. Conclusion: Dose or duration of thiopurine treatment did not explain a lack of increased risk of NHL with thiopurine use in Japanese patients with IBDs.

(C) 2022 The Author(s).

Published by S. Karger AG, Basel

\section{Introduction}

Immunomodulator therapy has been linked to an increased risk of malignancy in patients with inflammatory bowel diseases (IBDs) [1]. An approximately 4 -fold higher risk of lymphoma has been observed among patients treated with thiopurines in Western countries [2,3], with the risk increasing as the duration of thiopurine treatment is extended [4]. Combining anti-tumor necrosis factor a (anti-TNFa) therapy with thiopurines further elevates the risk of lymphoma [5]. Most studies of lymphoma risk in IBDs have been conducted in Caucasian populations and may not be generalizable to other populations, in whom the incidence of hematological malignancies, including non-Hodgkin lymphoma (NHL), is generally lower [6]. 
We have previously reported that treating IBDs with a thiopurine, with or without an anti-TNFa agent, did not increase the risk of NHL in a real-world study of Japanese patients with IBDs [7]. Although differences in genetic background and baseline incidence of NHL may explain the lack of increased risk of lymphoma with thiopurine and anti-TNFa therapy, the standard dose of thiopurines prescribed to Japanese patients with IBDs is typically lower than for Western patients [8] in association with the impacts of nudix hydrolase 15 polymorphisms. We therefore considered whether the difference in incidence of NHL in the Japanese population, relative to global study populations, is a consequence of differences in thiopurine dose. In this subgroup analysis, we investigated whether dose of thiopurine treatment influenced the risk of NHL in Japanese patients with IBDs, using data from a large national administrative database. The effect of age and disease type (ulcerative colitis [UC] or Crohn's disease $[\mathrm{CD}]$ ) on NHL incidence was also investigated.

\section{Methods}

We conducted this retrospective study, analyzing the incidence of NHL among Japanese patients with IBDs prescribed thiopurine treatment, using cross-sectional data from the private Medical Data Vision (MDV) claims database between 1 April 2008 and 31 January 2018, as previously reported [6]. At the time of the study, the MDV database contained hospitalization, outpatient, and prescription data for approximately 17.8 million patients treated at Diagnosis Procedure Hospitals in Japan.

Eligible patients received $\geq 1$ diagnosis of UC or CD (World Health Organization's International Statistical Classification of Diseases and Related Health Problems [10th ed.; ICD-10 codes K51 and K50, respectively] and no diagnosis of malignancy at the index date; this was defined as the first day of the month in which an IBD was first diagnosed or the date of first thiopurine prescription [azathioprine or 6-mercaptopurine], with or without an antiTNFa agent [infliximab or adalimumab]). Occurrences of NHL (ICD-10 codes C82, C83, C84, C85, and C86) were identified during the evaluation period (the period from the index date until the date of malignancy diagnosis or end of the observation period [31 January 2018]), according to the following criteria: $\geq 3$ diagnoses of NHL; $\geq 1$ diagnosis of NHL and a diagnostic imaging procedure; or $\geq 1$ diagnosis of NHL and $\geq 1$ prescription of rituximab, cisplatin, cyclophosphamide, doxorubicin, vincristine, cytarabine, etoposide, carboplatin, brentuximab vedotin, or ifosfamide.

This subgroup analysis evaluated incidence rate ratios (IRRs) for NHL by total dose prescribed, mean daily dose prescribed, duration of thiopurine treatment prescription, age, and disease (UC or CD). For all analyses, the study population was categorized according to treatment (thiopurine monotherapy; thiopurine and anti-TNFa therapy; and any thiopurine use). Age- and sex-adjusted IRRs and 95\% confidence intervals for NHL were calculated as the ratio of incident cases in the subgroups relative to the overall study population.

\section{Results}

During the study observation period, a total of 103 cases of NHL were recorded among 75,673 patients with IBDs. Of these cases of NHL, 41 received a thiopurine (21 received only thiopurine; 20 received thiopurine and antiTNFa therapy). As previously reported [7], there was no overall increase in the risk for developing NHL among Japanese patients treated with thiopurines. Specifically, the IRRs relative to the overall IBD population were 1.88 , 1.42 , and 0.38 for $<1$ year, $1-3$ years, and $\geq 3$ years of thiopurine treatment, respectively (Fig. 1). Likewise, IRRs for NHL relative to the overall IBD population were 1.65 , $2.06,1.04$, and 0.34 among patients prescribed a total thiopurine dose of $\leq 7.50 \mathrm{~g},>7.50-28.28 \mathrm{~g},>28.28-66.55 \mathrm{~g}$, and $>66.55 \mathrm{~g}$ (Fig. 2). There were no notable differences in NHL incidence when grouping patients by mean daily thiopurine dose prescribed (Fig. 2), age, or disease subgroups (online suppl. Fig. 1, 2; for all online suppl. material, see www.karger.com/doi/10.1159/000519560).

\section{Discussion}

Thiopurine treatment has consistently been associated with an increased risk of developing lymphoma in patients with IBDs $[2,3]$. A longer duration of thiopurine treatment [4] and combination treatment with an antiTNFa agent [5] are also known to exacerbate the risk of lymphoma. However, most studies reporting an increased risk of NHL with thiopurine or anti-TNFa treatment have been conducted in predominantly Caucasian populations with IBDs $[2,3]$. Among the few studies conducted in Japanese patients with IBDs, thiopurine use was not found to be a risk factor for hematological malignancies [7, 9]. However, the overall incidence of NHL is lower in Japanese than in Western populations [6], and Japanese patients are generally prescribed lower doses of thiopurines [8], which may partly explain the lack of an association between thiopurine treatment and lymphoma risk.

In this real-world study investigating NHL incidence among Japanese patients with IBDs using a large administrative claims database, a lack of increased risk for NHL with thiopurine treatment could not be explained by dose or duration of thiopurine treatment. Similarly, there were no notable differences in NHL incidence among age and disease (UC or CD) subgroups. Considering that no difference was seen in terms of the parameters age and daily dose, there is no signal of an increased risk with high dose 


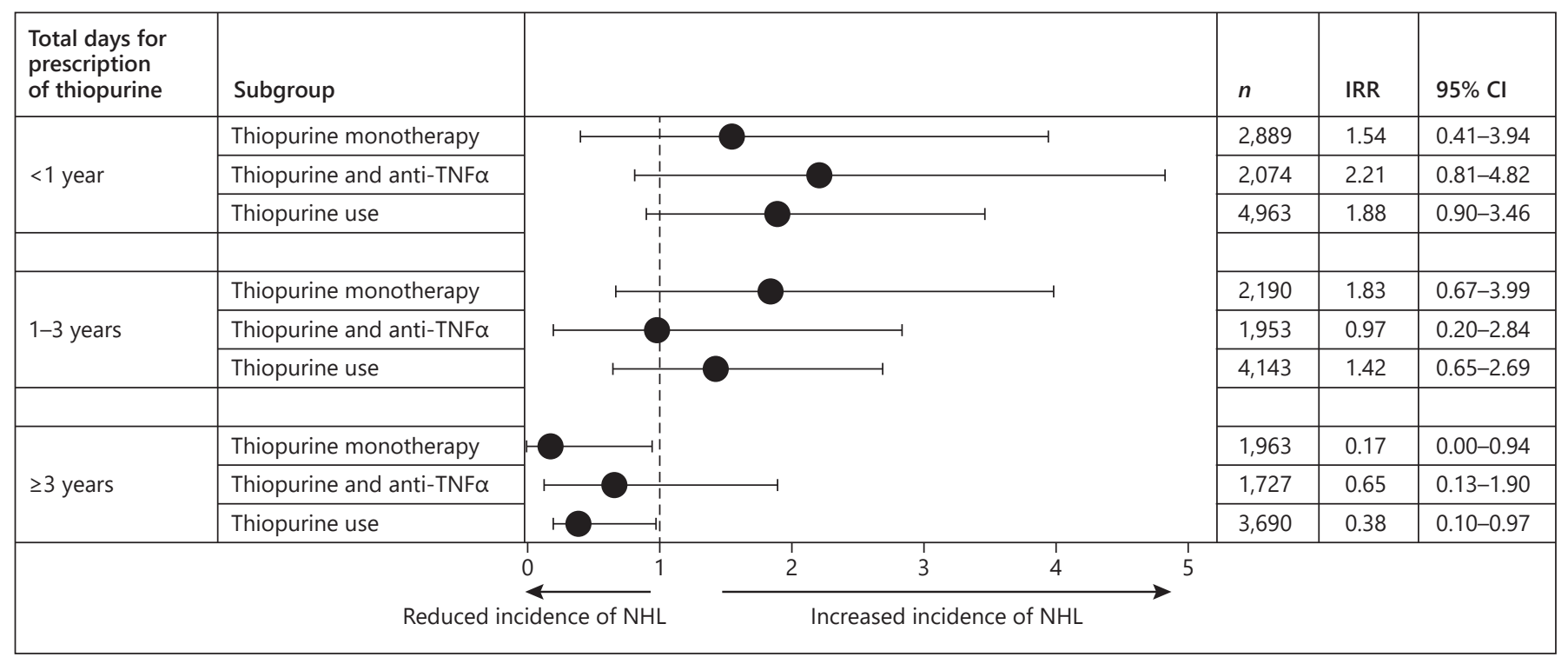

Fig. 1. Age- and sex-adjusted IRRs for NHL in thiopurine prescription duration subgroups of patients with IBDs, relative to the overall IBD study population, by treatment prescribed (MDV database). CI, confidence interval; IBD, inflammatory bowel disease; IRR, incidence rate ratio; MDV, Medical Data Vision; NHL, non-Hodgkin lymphoma; TNFa, tumor necrosis factor alpha.

and long duration of thiopurine treatment, even when these parameters are removed. However, we did observe a lower incidence of NHL with longer duration of thiopurine treatment ( $\geq 3$ years) and higher total dose of thiopurine prescribed $(>66.55 \mathrm{~g})$. This unexpected finding may reflect a tendency for patients with a long duration of (and therefore greater overall exposure to) thiopurine therapy to have achieved optimal disease control, without experiencing adverse events associated with excessive immunosuppression, including NHL, that result in treatment discontinuation. For example, high levels of inflammation have been linked to an increased risk of lymphoma in patients with rheumatoid arthritis [10]. Patients with high overall disease activity were 70 times more likely to present with lymphoma compared with patients with low disease activity, whereas no correlation between duration of azathioprine use and lymphoma incidence was observed [10]. Patients remaining on thiopurine treatment may therefore represent a subset of patients with an inherently lower risk for hematological complications because they have relatively low levels of well-managed inflammation compared with patients who switch treatments or have their treatment escalated. Furthermore, the relative increase in risk of NHL after short-term exposure ( 12 months) observed in this study is consistent with what has been reported elsewhere [3]. The potential reduction in the risk of NHL among patients with thiopurine therapy was only observed after a longer duration of follow-up in this study, although no change in risk was observed with duration of thiopurine treatment in patients with rheumatoid arthritis [10]. IRRs were higher in patients aged 20-29 years receiving thiopurine and anti-TNFa therapy compared with the overall group; however, considering this trend was not observed in close age ranges, it is uncertain whether this represents a true risk.

As this retrospective study utilized an administrative claims database, it is limited by potential information bias and confounding factors [7]. It is possible that diagnoses and procedures may have been incorrectly coded. Likewise, confounding factors for NHL could not be adjusted for as this information was not captured by the database. It is possible that there were "hidden" risk factors, such as the association of specific genotypes with thiopurine dose and treatment duration.

Patients were also not tracked longitudinally and filling a prescription does not guarantee medication adherence. Furthermore, the low number of incident cases of NHL among the IBD cohort limits the conclusions that can be drawn from these subgroup analyses. Finally, the generalizability of the study is likely limited to other East Asian populations with similar genetic backgrounds [8]. The balance between the benefits and risks of long-term thiopurine use in this patient population is different from 


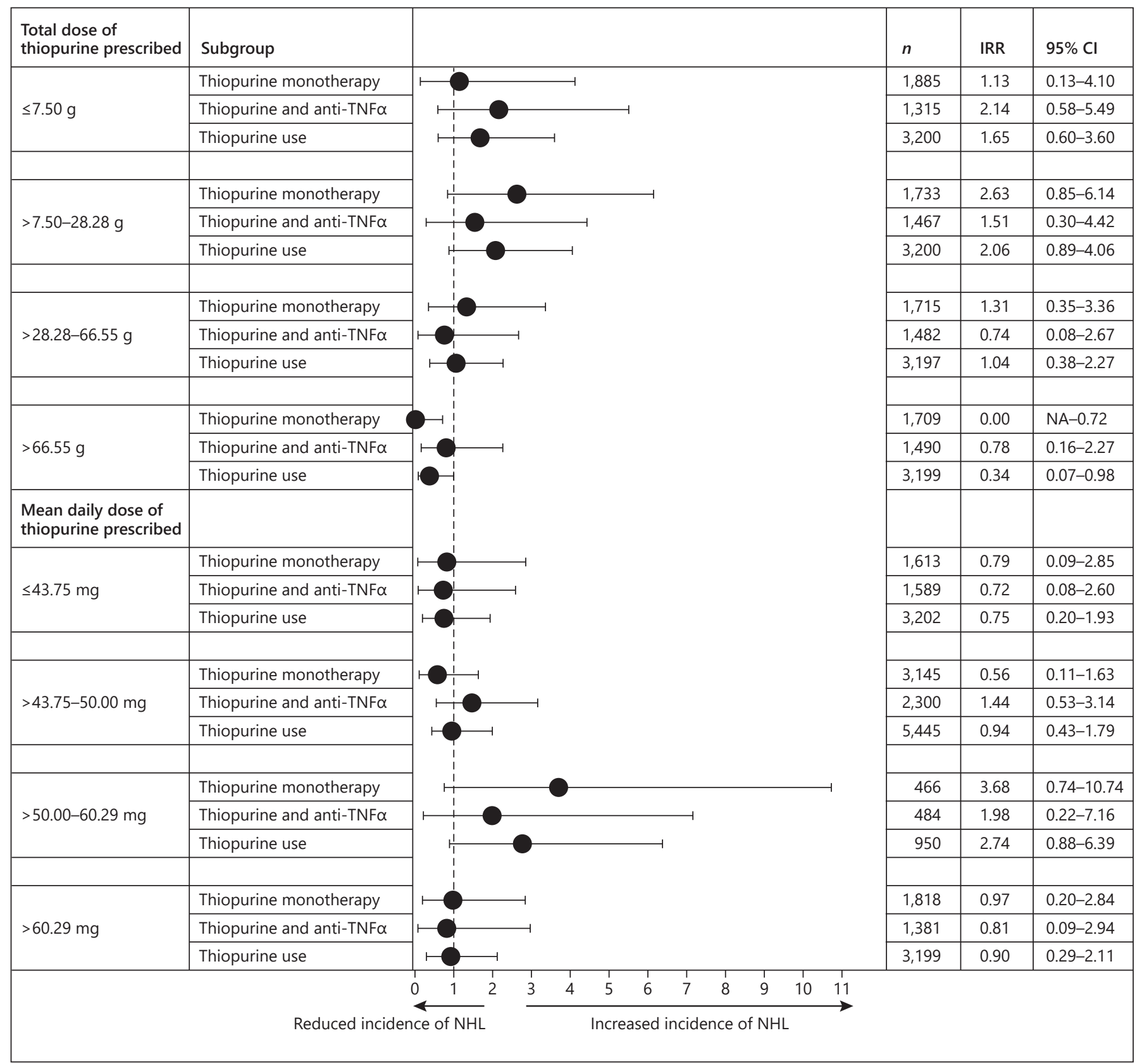

Fig. 2. Age- and sex-adjusted IRRs for NHL in total and mean daily dose of thiopurine prescription subgroups among patients with IBDs, relative to the overall IBD study population, by treatment prescribed (MDV database); dose levels have been rounded to 2 decimal places. CI, confidence interval; IBD, inflammatory bowel disease; IRR, incidence rate ratio; MDV, Medical Data Vision; NA, not available; NHL, non-Hodgkin lymphoma; TNFa, tumor necrosis factor alpha.

that previously reported in the Western population. Further studies are needed to understand how additional confounding factors influence NHL risk with immunosuppressive treatments. In conclusion, dose or duration of thiopurine treatment did not explain a lack of increased risk of NHL with thiopurine use in Japanese patients with IBDs in this real-world retrospective study conducted using a large, administrative claims database. 


\section{Acknowledgments}

Support for the data analysis was provided, under the direction of the authors, by Sachie Inoue, $\mathrm{PhD}$, an employee of Crecon Medical Assessment. The authors would also like to thank Kosuke Iwasaki, $\mathrm{PhD}$, and Wentao Tang, $\mathrm{PhD}$, employees of Milliman, and Akihito Uda, PhD, for developing the concept (WT and AU) and analyzing data (KI). Medical writing support was provided by Marissa Scandlyn PhD CMPP and Ben Searle PhD of MIMS (Hong Kong) Ltd. and funded by Takeda Pharmaceutical Co. Ltd.

\section{Statement of Ethics}

The study was conducted according to the Declaration of Helsinki and the International Society for Pharmacoepidemiology Guidelines for Good Pharmacoepidemiology Practices. It was carried out retrospectively using a database of anonymized data, following Ethical Guidelines for Medical and Health Research Involving Human Subjects issued by the Japanese Ministry of Health, Labor, and Welfare [11]. The investigators could only access anonymized information from MDV database; therefore, in accordance with the Japanese Ethical Guidelines mentioned above, institutional ethics approval and informed consent were not required.

\section{Conflict of Interest Statement}

T.K. reports personal fees from Astellas, Celltrion, Covidien, Eli Lilly, Ferring, Gilead Sciences, Janssen, JIMRO Co., Ltd, Mitsubishi Tanabe Pharma, Pfizer, Takeda Pharmaceutical Company Limited, grants from Otsuka Holdings, and grants and personal fees from AbbVie GK, Alfresa Pharma, EA Pharma Co., Ltd, Kyorin Pharmaceutical Co. Ltd, Mochida Pharmaceutical Ltd, Nippon
Kayaku, Thermo Fisher Scientific, ZERIA Pharmaceutical, outside the submitted work. E.U. was an employee of Takeda Pharmaceutical Co. Ltd. during the conduct of the study. T.H. reports personal fees from AbbVie GK, Aspen Japan K.K., Bristol-Myers Squibb, Celltrion, Eli Lilly, Ferring, Gilead Sciences, Janssen, Kissei Pharmaceutical, Mitsubishi-Tanabe Pharma, Nichi-Iko Pharmaceutical, Nippon Kayaku, Pfizer, Takeda Pharmaceutical Company Limited, grants from AbbVie GK, EA Pharma, Otuska Holdings, and grants and personal fees from JIMRO, Kyorin, Mochida Pharmaceutical, Zeria Pharmaceuticals, outside the submitted work.

\section{Funding Sources}

This work was supported by Takeda Pharmaceutical Company Limited.

\section{Author Contributions}

All authors have made substantial contributions to all of the following: (1) the conception and design of the study, or acquisition of data, or analysis and interpretation of data, (2) drafting the article or revising it critically for important intellectual content, and (3) final approval of the version to be submitted.

\section{Data Availability Statement}

The data that support the findings of this study are available from MDV Co., Ltd. but were used under license for the current study; therefore, restrictions apply, and the data are not publicly available. Enquiries regarding the data can be directed to the corresponding author.

\section{References}

1 Axelrad JE, Roy A, Lawlor G, Korelitz B, Lichtiger S. Thiopurines and inflammatory bowel disease: current evidence and a historical perspective. World J Gastroenterol. 2016 Dec;22(46):10103-17.

2 Kandiel A, Fraser AG, Korelitz BI, Brensinger $\mathrm{C}$, Lewis JD. Increased risk of lymphoma among inflammatory bowel disease patients treated with azathioprine and 6-mercaptopurine. Gut. 2005 Aug;54(8):1121-5.

3 Lemaitre M, Kirchgesner J, Rudnichi A, Carrat F, Zureik M, Carbonnel F, et al. Association between use of thiopurines or tumor necrosis factor antagonists alone or in combination and risk of lymphoma in patients with inflammatory bowel disease. JAMA. 2017 Nov;318(17):1679-86.

4 Khan N, Abbas AM, Lichtenstein GR, Loftus EV Jr, Bazzano LA. Risk of lymphoma in patients with ulcerative colitis treated with thiopurines: a nationwide retrospective cohort study. Gastroenterology. 2013 Nov;145(5): 1007-15.e3
5 Chupin A, Perduca V, Meyer A, Bellanger C, Carbonnel F, Dong C. Systematic review with meta-analysis: comparative risk of lymphoma with anti-tumour necrosis factor agents and/ or thiopurines in patients with inflammatory bowel disease. Aliment Pharmacol Ther. 2020 Oct;52(8):1289-97.

6 Chihara D, Ito H, Matsuda T, Shibata A, Katsumi A, Nakamura S, et al. Differences in incidence and trends of haematological malignancies in Japan and the United States. Br J Haematol. 2014 Feb;164(4):536-45.

7 Kobayashi T, Uda A, Udagawa E, Hibi T. Lack of increased risk of lymphoma by thiopurines or biologics in Japanese patients with inflammatory bowel disease: a large-scale administrative database analysis. J Crohns Colitis. 2020 Jun;14(5):617-23.
8 Kakuta Y, Kinouchi Y, Shimosegawa T. Pharmacogenetics of thiopurines for inflammatory bowel disease in East Asia: prospects for clinical application of NUDT15 genotyping. J Gastroenterol. 2018 Feb;53(2):172-80.

9 Dulai PS, Siegel CA. The risk of malignancy associated with the use of biological agents in patients with inflammatory bowel disease. Gastroenterol Clin North Am. 2014 Sep; 43(3):525-41.

10 Baecklund E, Iliadou A, Askling J, Ekbom A, Backlin C, Granath F, et al. Association of chronic inflammation, not its treatment, with increased lymphoma risk in rheumatoid arthritis. Arthritis Rheum. 2006 Mar;54(3): 692-701.

11 Ministry of Health Labour and Welfare. Ethical guidelines for medical and health research involving human subjects. 2015 [cited 2021 Apr 1]. https://www.mhlw.go.jp/file/06-Seisakujouhou-10600000-Daijinkanboukouseikagakuka/0000080278.pdf.
Thiopurine Treatment in IBD with No Increased Lymphoma Risk 